\title{
Quantifying the Value of Emotions Using a Willingness to Pay Approach
}

\author{
Hi Po Bobo Lau $\cdot$ Mathew P. White $\cdot$ Simone Schnall
}

Published online: 12 October 2012

(C) The Author(s) 2012. This article is published with open access at Springerlink.com

\begin{abstract}
People generally seek out positive moods and avoid negative moods; however, it is unclear which motivation is more pronounced. Two studies addressed this issue by developing a value-based ranking of emotions based on the willingness to pay (WTP) approach. The approach utilizes money's cardinal properties and assumes opportunity costs as with everyday purchases. In Study 1 British participants indicated they would be willing to pay more to experience positive than to avoid negative emotions. In Study 2 this positivity bias was replicated with another sample of British participants. However, Hong Kong Chinese participants did not show such a preference, and were willing to pay significantly less to experience positive emotions but more to avoid negative emotions when compared with British participants. Experiencing Love was given the highest WTP judgment in all samples. Thus, some emotions are universally valued, whereas preferences for others differ across cultural groups, perhaps shaped by norms. Implications concerning valuations of psychological states for policy purposes are discussed.
\end{abstract}

Keywords Subjective well-being - Emotion - Affect - Willingness to pay · Culture · Affective forecasting

\section{Introduction}

Aristotle argued that the accumulation of wealth is not an end in itself, but only a means to achieving the real end: Happiness (Aristotle, 350 B.C./1988). Psychological research supports this contention (Diener and Lucas 2004). For instance, people say they save

H. P. B. Lau

University of Hong Kong, Pokfulam, Hong Kong

M. P. White $(\bowtie)$

European Centre for Environment and Human Health (ECEHH), University of Exeter Medical School, Knowledge Spa, Royal Cornwall Hospital, Truro TR1 3HD, UK

e-mail: mathew.white@exeter.ac.uk

S. Schnall

University of Cambridge, Cambridge, UK 
money to achieve future emotional well-being (Canova et al. 2005). We pay for a holiday to experience positive emotions such as joy and calm, and buy holiday insurance to avoid negative emotions such as worry and regret. The aim of the current research was to examine just how much money people are willing to pay to experience different positive emotions and avoid negative ones. For reasons developed below, we focus on direct emotional experiences unmediated by the purchase of goods and services usually associated with attempts to achieve or avoid emotional states. In our opinion, the application of economic methods developed in the context of valuing different states of the world to valuing different emotional states can illuminate a number of significant issues regarding well-being.

Although some research has explored the relative impact of actual experiences of positive and negative emotions on assessments of global well-being (e.g., Kuppens et al. 2008; Suh et al. 1996), we know relatively little about which emotions people think will have the most influence, and as a consequence, they are more likely to pursue or avoid. Kahneman (2000) refers to this distinction as one between experienced utility, namely the emotions actually experienced, and decision utility, namely the choices made on the basis of predicted emotional experiences. This distinction is critical because there are often discrepancies between the intensity and duration of what people expect to feel, which influences their decisions and choices, and what they actually do feel once a choice has been made (Wilson and Gilbert 2003). In the current research we explore this issue by examining whether people are prepared to pay a premium for experiencing positive or avoiding negative emotions, and the degree to which such preferences are consistent with existing literature on the likely impact of differently valenced emotions on global well-being.

The approach of putting a price tag on emotional experiences may also provide insight into cross-cultural differences regarding the desirability of emotional experiences. Several studies have asked participants from different cultures about the degree to which positive and negative emotions are culturally acceptable, ideal, and desirable. Despite some withinculture heterogeneity, respondents in collectivist cultures tend to be more accepting of negative emotions than those in individualistic ones (Eid and Diener 2001; Sommers 1984a; Tsai et al. 2006). More nuanced examinations of specific emotions, however, are restricted by the use of traditional Likert-type scales that fail to incorporate the kinds of trade-offs and opportunity costs involved in decision utility. For instance, respondents could consider both fear and anger "very undesirable," but this leaves unclear which emotion they would prefer to avoid if given a choice.

\subsection{The Contribution of Positive and Negative Emotions to Well-Being}

Subjective well-being (SWB) is defined in terms of how people think and feel about their lives (Diener et al. 1999), and is considered high when positive thoughts and feelings outweigh negative ones, and low for the opposite pattern. Kahneman and colleagues have argued that this approach to well-being has its origins in Utilitarian definitions of happiness and, as envisaged by these philosophers, could play an important role in improving policyrelated resource allocation decisions (Dolan and Kahneman 2008; Kahneman et al. 2004; Kahneman and Sugden 2005).

However, in the search for greater SWB is it better to pursue positive emotions or avoid negative ones? Kahneman and Tversky (1979) are among those who argue that negative stimuli tend to influence us more than positive ones, and thus it makes sense to focus on reducing negative emotions (e.g. Baumeister et al. 2001, although see Schimmack 2005). Others recognize the unique contribution of positive emotions (e.g., Fredrickson 2004), not least in terms of reducing morbidity and mortality (Cohen and Pressman 2006; Danner 
et al. 2001). Importantly, positive and negative emotions are not simply two ends of the same spectrum but constitute two related yet separate dimensions (Cacioppo et al. 1999), and are associated with different neural architectures in the brain (Davidson et al. 2000). Thus, the relative importance of negatives and positives for overall well-being becomes an important issue.

A number of studies by Diener and colleagues have investigated this question (Kuppens et al. 2008; Oishi et al. 2007; Suh et al. 1996; Suh et al. 1998), but have yielded somewhat equivocal results. Suh et al. (1996) asked US students to recount whether they had experienced a number of positive and negative life events (e.g. made a new friend vs. gained weight) within the previous 4 years. Positive and negative events correlated equally strongly with current life satisfaction. A follow-up study across 41 nations using the World Values Survey similarly found that both positive and negative affect showed comparable correlations with life satisfaction (Suh et al. 1998). A second study with college students from 40 nations, however, indicated a stronger influence of positive than negative affect on life satisfaction (Suh et al. 1998).

Oishi et al. (2007) extended this work and also examined the relationship between life satisfaction and daily positive and negative events across different cultures. For European Americans each negative event had nearly twice the impact in satisfaction as each positive event, which contrasts with Suh and colleagues' (1996) finding that positive and negative affect had roughly the same impact. For Asian Americans and Koreans negative events had slightly more impact and for Japanese students they had the same impact as positive ones, more in line with Suh et al. (1996). Thus, an important contribution of Oishi and colleagues (2007) is the recognition of cultural differences in the relative importance of negative and positive events and emotions for overall life satisfaction.

Extending this work further, Kuppens et al. (2008) looked at life satisfaction data from nearly nine thousand people from 46 different countries. Averaging across all countries, and in contrast to Suh et al. (1996) and Suh et al. (1998, Study 1), but consistent with Suh et al. (1998, Study 2), they found that positive emotions had nearly twice the influence on life satisfaction as negative emotions. Again, however, and in line with Oishi et al. (2007), this overall effect was moderated by culture, such that negative emotions were more important in predicting life satisfaction in individualistic cultures like the US rather than collectivist cultures like China.

\subsection{Preferences and Norms for Emotion Experiences}

In addition to the actual contribution of different emotional experiences to people's wellbeing, "ideal affects" have been construed as emotional goals that people strive for through their daily activities (Larsen 2000; Tsai et al. 2006). Not surprisingly, most people report that their ideal emotional states are pleasant, rather than unpleasant (Augustine et al. 2010; Kampfe and Mitte 2009). Nevertheless, noteworthy moderating factors such as cultural background (Tsai et al. 2006), individual differences such as level of extraversion (Rusting and Larsen 1995) and interpersonal goals (Tsai et al. 2007) have been observed. Discrepancies between these ideal emotional states and actual emotional states incur psychological cost such as depression (Tsai et al. 2006) and lowered life satisfaction (Kampfe and Mitte 2009). If we consider people's actions as habitual attempts to regulate discrepancies between ideal and actual emotions, knowing which emotions people desire and value will help us understand why people engage in certain emotion-inducing behaviors but not others (Larsen 2000; Kampfe and Mitte 2009). 
Eid and Diener (2001) assessed participants' desirability of four positive (affection, joy, pride, and contentment) and four negative (anger, fear, sadness, and guilt) emotions. In the U.S., $83 \%$ percent of respondents rated all positive emotions as desirable and $44 \%$ rated all negative emotions as undesirable. In China, by contrast, only $9 \%$ of respondents thought all positive emotions were desirable and only $14 \%$ thought that all negative emotions were undesirable. Thus, whereas American participants generally saw positive emotions as desirable, and negative emotions as undesirable, Chinese participants preferred more of a balance of both.

Eid and Diener (2001) further noted the rather homogeneous norm for Western participants to feel good (see also Sommers 1984b). Other findings also support this notion of a cultural expectation of positive affect. Sommers (1984b) showed that American participants considered positive feelings more typical to occur on a daily basis, both for themselves, and for the average person. In addition, other people were rated as more likeable if they were thought to generally show more positive than negative affect. Further, although Asians tend to report fewer positive experiences than Western participants, this bias seems to be due to fewer recalled positive events, rather than fewer experienced events (Oishi 2002; Wirtz et al. 2009). Thus, it appears that Western participants are well aware of the "(...) pressure to be joyful, happy, and full of love and pride and to make use of their constitutional right to the pursuit of happiness" (Eid and Diener 2001, p. 880), which might be reflected in their desired emotional states.

\subsection{Evaluating Emotional Preferences by Price-Tags}

To date, ideal emotional states have been measured by scales asking participants how much they wish to feel or avoid certain positive emotions and negative emotions (e.g., Augustine et al. 2010; Kampfe and Mitte 2009; Rusting and Larsen 1995; Tsai et al. 2006). However, it may also be beneficial to explore what people are prepared to do to achieve the most desirable emotions. Are they willing to spend effort, time and even money to pursue them?

Economists have long studied individuals' likes and dislikes through their revealed and stated preferences (e.g., Adamowicz et al. 1994). Revealed preference operationalizes individuals' preferences for options by looking at what they chose. For example, by assessing which dress the consumer has purchased, we know which dress she prefers, and how valuable it is to her. When goods are not openly traded in the market and preferences cannot be revealed though purchasing behavior, the alternative approach of stated preferences has been adopted (Kahneman et al. 1993). For instance, people are asked how much money they would be willing to pay to experience some good (e.g. a park near their house) or how much compensation they would be willing to accept to have some bad imposed upon them (e.g. building on a nearby park). Such stated preferences are strongly related to predicted satisfaction with specific interventions (Kahneman et al. 1993).

As non-market goods, emotional experiences could be quantified using a similar strategy. Compared to Likert scale ratings, WTP judgments offer the following advantages as a metric for evaluation. First, the approach assumes opportunity costs behind purchases. With limited money and time, every activity has a trade-off: By buying the sandwich, we forgo the opportunity to spend that sum on the pasta for lunch; by attending the lecture, we forgo the opportunity to spend time with friends. When participants assign a price to an emotional experience, they have decided to forgo the opportunity to spend that amount of money on alternative purchases. In contrast, Likert scales do not capture the trade-offs between purchases that are implied in this pricing exercise. Second, the use of monetary 
values to quantify emotional experience allows researchers to exploit the ratio property of money (i.e., USD 5 is half of USD 10) and enables comparisons between emotional experiences with different ranks based on their means derived from Likert scales. For instance, besides testing whether happiness is valued more highly than pride, we can quantify this preference. The WTP approach may facilitate the study of emotional experience by making participants' emotional experiences comparable with their daily consumption behavior, because the same metric is used for emotional experiences as for hourly wages, a family dinner, or a favorite pair of jeans.

\subsection{The Current Research}

Our goal was to develop a value-based ranking of different emotions by having people assign hypothetical monetary values to time-limited emotional states. Building on the logic of using WTP estimates for valuations of non-market goods, participants were asked to state the amount they would be prepared to pay to experience a range of specific positive emotions (e.g. happiness, love) and avoid specific negative emotions (e.g. fear, anger) for a limited time. Study 1 tested British participants. In Study 2, we aimed to replicate the pattern of the value-based ranking of emotions of British participants obtained in Study 1 using a more comprehensive repertoire of emotion items. Moreover, because previous research (e.g., Tsai et al. 2006; Eid and Diener 2001) suggested that individualistic and collectivistic cultures differ in the emotional experiences they see as normative and desirable, we investigated how value-based rankings of emotions vary across cultural groups, and compared British students to Chinese students in Hong Kong. Overall, the goal was to explore how people decide to spend one of their main limited resources, namely money, in order to maximize well-being and happiness.

\section{Study 1}

This study investigated whether British participants are willing and able to differentiate between different emotional experiences via a WTP approach. Given that people may find this exercise unusual we contextualized it by also asking how much they would be willing to pay to experience familiar positive and negative activities.

We deemed two outcomes possible. On the one hand, based on the literature suggesting that negative emotions and events factor more heavily into people's well-being (Oishi et al. 2007), participants might prefer avoiding negative emotions over experiencing positive ones. On the other hand, because experiencing positive affect in individualistic societies is highly normative (Eid and Diener 2001; Sommers 1984b), the opposite pattern might be obtained. Indeed, Tsai et al. (2006) note that cultural norms influence ideal affect more strongly than actually experienced affect. Thus, participants might follow the uniform norms identified by Eid and Diener (2001), which for individualistic cultures stipulate a strong expectation to experience positive emotions, such as happiness, love and pride.

\subsection{Methods}

\subsubsection{Participants}

Ninety-seven students (17 male) from the University of Plymouth who identified "British" as their nationality participated in an unpaid online survey in response to email invitations sent via student email lists. 


\subsubsection{Materials and Procedure}

A survey was designed to measure participants' WTP judgments for re-creating positive emotional experiences (love, happiness, and pride) or avoiding negative emotional experiences (anger, embarrassment, fear, disgust, loneliness, worry, guilt, regret, sadness, and nervousness). Participants were asked to imagine that each emotional episode was of high intensity and would last for $1 \mathrm{~h}$. Prior to giving their WTP judgments, participants were asked to consider an episode of a specific emotional experience that included some of the appraisals (Ortony et al. 1988) associated with a given emotion. For happiness, for instance, participants were instructed:

Think of a specific time when you were very happy. This might have been because you felt very content with a specific situation in your life. Think of the feeling you experienced at the time. How much would you be willing to pay to re-create this feeling for one hour?

Participants then indicated a price between GBP 10 and GBP 150, with GBP 10 increments. Additionally, participants were asked to assign prices to four events: Spending time with a person they care about, engaging in a favorite task, avoiding a person they dislike, and avoiding a disliked task. These experiences were also said to be $1 \mathrm{~h}$ long and were answered on the same scale. Data were collected via an online survey during Fall 2007. Informed consent was sought prior to the start of the survey, and debriefing was provided upon completion. The study was approved by the University of Plymouth's School of Psychology Research Ethics Committee.

\subsection{Results}

\subsubsection{Do People Give Different WTP Judgments for Different Emotional Experiences?}

We first tested whether people assigned different prices to experience positive emotions and avoid negative ones. We aggregated WTP judgments for experiencing love, happiness, and pride to form a mean WTP score for positive emotions, and WTP for avoiding sadness, worry, guilt, embarrassment, loneliness, fear, regret, anger, nervousness, and disgust to form a mean WTP score for negative emotions. Table 1 presents the descriptive statistics and the ranks of the thirteen individual emotions and valence aggregates. Means and standard deviations are in Pound Sterling. A paired-samples $t$ test suggested that participants were willing to pay more to experience positive emotions than to avoid negative emotions, $t(96)=8.13, p<.001, d=0.47$. For individual emotions, experiencing love was ranked first, followed by happiness, and avoiding sadness and worry.

Repeated contrasts suggested that the WTP of experiencing happiness was higher than for avoiding sadness, $F(1,88)=6.42, p<.01$ (and all subsequent emotions), that avoiding sadness was higher than avoiding worry, $F(1,88)=5.09, p<.02$ (and all subsequent emotions), and avoiding nervousness was higher than avoiding disgust, $F(1,88)=9.60$, $p<.003$ (and all subsequent emotions). In other words, participants were keener to experience positive emotions than to avoid negative ones, as reflected in their WTP judgments.

\subsubsection{Does the Positivity Bias Extend to More Familiar Scenarios?}

To ensure that the emotion-related results were not simply a function of the unfamiliarity of the task we also evaluated participant's WTP to engage in positive versus negative 
Table 1 Study 1: means and standard deviations of willingness to pay judgments for different emotional experiences (ranked from highest to lowest)

\begin{tabular}{lll}
\hline Emotional experiences & Mean WTP (in GBP) & SD \\
\hline Love & 95.26 & 50.00 \\
Happiness & 89.05 & 47.74 \\
Sadness & 81.58 & 51.43 \\
Worry & 68.97 & 48.06 \\
Guilt & 66.15 & 44.52 \\
Embarrassment & 63.40 & 43.37 \\
Loneliness & 63.40 & 46.97 \\
Regret & 62.37 & 46.43 \\
Fear & 61.68 & 40.96 \\
Anger & 59.28 & 46.55 \\
Pride & 59.18 & 46.41 \\
Nervousness & 59.17 & 46.87 \\
Disgust & 43.20 & 35.25 \\
Mean positive & 81.05 & 42.97 \\
Mean negative & 62.84 & 34.91 \\
\hline
\end{tabular}

activities, and spend time with liked vs. disliked persons using a 2 (Valence: Positive, Negative) $\times 2$ (Type of experience: People, Task) repeated-measures ANOVA. Participants gave higher WTP judgments for re-creating pleasant experiences, (Liked person: $M=99.89, \mathrm{SD}=48.54$; Favorite task: $M=51.05, \mathrm{SD}=41.53$ ) than for avoiding unpleasant ones (Disliked person: $M=38.53, \mathrm{SD}=32.78$, Disliked task: $M=26.95$, $\mathrm{SD}=27.83), F(1,94)=114.79, p<.001$. Spending time with people elicited higher WTP evaluations in general than activities, $F(1,94)=94.23, p<.001$ (means above), and the significant interaction between valence and type of experience, $F(1,94)=33.29$, $p<.001$, suggests that people elicited greater polarization in WTP evaluations for liked versus disliked people than pleasant versus unpleasant activities. To summarize, the positive-negative asymmetry observed for emotional experiences extended to time spent with people and activities.

\subsection{Discussion}

Study 1 was a first attempt to apply the WTP approach to understand people's emotional preferences. Results suggest that participants are willing and able to differentiate emotions through different WTP judgments to arrive at a ranking of emotional experiences. Participants gave higher WTP judgments to experience positive emotions than to avoid negative ones.

Our findings are consistent with earlier suggestions that in individualistic cultures it is highly normative to experience positive affect (e.g., Eid and Diener 2001; Sommers 1984b), and thus, our findings might reflect participants' awareness that in their cultural context, pursuing positive affect is both normative and desirable. Ironically however, as noted by Oishi et al. (2007), in a culture that highly prizes positive affect, and where good moods are prevalent most of the time, a given negative event can be much more detrimental to overall life satisfaction (although see Kuppens et al. 2008, for different results). In this sense, participants may be showing an error of affective forecasting (Wilson and 
Gilbert 2003), because they do not recognize the actual benefit of avoiding negative emotions over positive emotions.

Participants' greater preference for experiencing positive than avoiding negative emotions extended to time spent on liked and disliked events and people, such that participants were willing to pay the most for time spent with their favorite person. Indeed, the values assigned to an hour with a favorite person and an hour of experiencing love were almost identical (GBP 100 vs. GBP 95), supporting our contention that the WTP method of valuing emotions was readily understood by participants. Our pattern of findings is in line with previous findings on the importance of love relative to other positive emotional experiences such as joy and pride across cultures (Sommers 1984a), and the contribution of belongingness to psychological well-being (Baumeister and Leary 1995; Deci and Ryan 2000; Ryff 1989).

Despite this convergence our results differed from those of Eid and Diener (2001), who used a Likert scale approach. First, whereas they found pride to be a highly valued emotion, our respondents were not willing to pay much to experience it compared to other positive emotions. One interpretation of this difference may be that people feel that pride is something you have to earn yourself and that cannot be bought, or else it becomes false pride. Another possibility is that the British may not see pride as a particularly desirable emotion. Sommers (1984a), for instance, found that $47 \%$ of her (small) American sample thought pride was a constructive emotion but that only $33 \%$ of Greeks and $25 \%$ of Chinese did, suggesting wide cultural variation in reactions to this particular emotion. Second, Eid and Diener (2001) found that sadness was viewed as more desirable than anger and fear. This would suggest that people should be willing to pay less to avoid sadness, but we found the opposite, suggesting that people do want to avoid it. The literature on psychological resilience highlights the benefits of some sadness and adversities in one's growth (Seery et al. 2010). However, such benefits are usually not witnessed at the time a loss is incurred, and sadness due to loss is more intense and dreadful to endure, compared to other negative emotions such as anger (Reisenzein 1994). It is therefore not hard to imagine that participants were willing to pay generously to sidestep the dolorous experience when asked to imagine encountering sadness in our WTP paradigm, but agreed that the experience is appropriate or even desirable in a preference paradigm such as Eid and Diener's (2001).

Encouraging as the results are, the positivity bias we observed may be attributed to the unequal salience of the positive and negative emotion items we used. Although we attempted to control the intensity of emotions by stating that each emotional experience would last for $1 \mathrm{~h}$ of high intensity, we had only three positive, but ten negative emotions, and thus the findings may be due to this difference. Study 2, therefore, used equal numbers of positive and negative emotions.

\section{Study 2}

Study 2 extended Study 1 in a number of ways. A limitation of research comparing the relative importance of positive versus negative emotions is that it does not tell us which specific emotions are best (or worst) for overall well-being. Many authors argue that a simple focus on valence can obscure important differences across specific emotions. For instance, appraisal theorists (e.g., Ortony et al. 1988; Roseman et al. 1990; Scherer et al. 2001) presume that specific emotions are the result of cognitive interpretations of emotioneliciting stimuli that produce adaptive responses. Thus, although happiness is considered a 
positive emotion, and anger a negative emotion, both involve approach tendencies and can lead to the same effects on cognitive processing (Bodenhausen et al. 1994), presumably because happiness and anger both imply that one can trust one's own inclinations (Clore and Huntsinger 2007). In terms of overall well-being we might therefore expect that people prefer to experience anger over other negative emotions, such as sadness or fear, and this is indeed what we found in Study 1. A similar logic might be applied to other pairs of negative or positive emotions that despite the same valence involve different appraisals and action tendencies.

Another aspect of emotion we take into account in the second study is arousal level. According to the circumplex model of emotion (Russell 1980), some emotions involve high arousal (e.g., excitement, enthusiasm), whereas others involve lower arousal (e.g., calm, boredom), and this study had more examples of each kind.

In Study 1 we had observed a general preference for positive emotions in British participants. Because Asians take into account both recalled positive and negative emotional moments when judging the desirability of life situations such as vacations (Wirtz et al. 2009), we predicted that such a positivity bias would be absent among Hong Kong Chinese participants. Further, A. Y. Lee et al. (2000) postulate that collectivism is closely tied to a prevention-oriented regulatory focus, and thus, Hong Kong Chinese participants may be willing to pay to prevent negative emotions compared to experiencing positive ones.

To help anticipate possible findings regarding specific emotions, we were once again guided by Eid and Diener (2001). Their respondents across cultures did not differ regarding the importance of affection, joy and contentment. However, collectivistic cultures perceived pride as more undesirable than their individualistic counterparts. The inappropriateness of pride resonates with the primacy of maintaining group harmony in collectivistic people's daily lives. Following a similar logic, we predicted that compared to British participants, Hong Kong Chinese participants would value the presence of happiness and pride less, but the absence of embarrassment more.

\subsection{Methods}

\subsubsection{Participants}

Eighty-seven participants were recruited via email invitations dispatched across student forums and university-wide email systems. Roughly half $(n=41)$ were from the University of Cambridge and identified themselves as British (14 male, mean age $=22.27$ ), whereas the remainder $(n=46 ; 21$ male, mean age $=21.13)$ were from the Hong Kong University of Science and Technology and identified themselves as Hong Kong Chinese. Because Hong Kong Chinese undergraduates are brought up in a multi-cultural, bilingual society with a strong English language heritage and must pass a public examination on academic English before entering university, an English questionnaire was employed among both Hong Kong Chinese and British undergraduates. This provided us with the opportunity to present the questionnaire to different cultural groups in exactly the same language. Advantages of this included linguistic continuity and lack of translation/interpretation issues.

\subsubsection{Materials and Procedure}

As in Study 1, the survey measured participants' WTP judgments for experiencing positive emotions or avoiding negative emotions that were specified to be of high intensity and to 
last for $1 \mathrm{~h}$. Positive emotions included love, pride, happiness, delight, excitement, calm and enthusiasm, and negative emotions included sadness, regret, embarrassment, frustration, fear, anger and boredom. British participants indicated a price between GBP 10 pounds to GBP 150, with GBP 10 increments, whereas Hong Kong Chinese participants indicated a price between HKD 100 to HKD 1,500, with HKD 100 increments. ${ }^{1}$

Data were collected from an internet survey server during Fall 2009. Informed consent was sought prior to the start of the survey, and debriefing was dispatched upon completion. The study was approved by the University of Cambridge's Social and Developmental Psychology Research Ethics Committee.

\subsection{Results}

\subsubsection{Replicating Study 1}

We first tested whether the positivity bias obtained in Study 1 was replicated in the current sample of British undergraduates. The judgments for the seven positive emotions and seven negative ones were averaged to form mean WTP judgments for positive and negative emotions respectively (Table $2^{2}$ ). A paired-samples $t$ test suggested that the mean WTP for positive emotions was higher than that for avoiding negative emotions, $t(40)=2.15$, $p<.04, d=0.22$. This pattern replicates Study 1 but the effect size now, with equal numbers of positive and negative emotions, was smaller. For the eight emotions that were assessed in both studies (love, sadness, happiness, embarrassment, regret, pride, anger, and fear), the rank order was largely replicated: Love was given the highest value, followed by happiness, sadness, fear, embarrassment, regret, pride and anger. The differences were that embarrassment and regret were above fear in Study 1, but just below it in Study 2, and whereas pride was below anger in Study 1, the order was reversed in Study 2. This general replication of the value-based ranking of emotions and the positivity bias suggests that the WTP approach may be a robust method of capturing emotion preferences.

\subsubsection{Do British and Hong Kong Chinese Participants Differ in Their Willingness to Pay for Different Emotions?}

A paired-samples $t$ test was used to compare the mean WTP for positive and negative emotions among Hong Kong Chinese participants. This time there was no significant difference, $t(45)=1.14, p=.26, d=0.12$. Nevertheless, like British participants, Hong Kong Chinese participants were willing to pay most for an hour of love and least to avoid an hour of boredom (Table 2). The rank orders for the emotions between these extremes were quite different across cultures. For instance, although happiness was ranked second

\footnotetext{
1 Purchasing power parity factors of GBP and HKD were taken into consideration in creating equivalent scales. Purchasing power parity indicates the amount of a certain currency required to purchase USD 1 worth of goods in a given country, and thus allows currency conversions that eliminate national differences in price levels (United Nations Statistics Division 2009). At the time of the survey, GBP 1 equated HKD 11.8. However, for ease of administration of the survey, we converted GBP 1 to HKD 10 when designing the scale.

${ }^{2}$ Unexpectedly, WTP judgments of British participants in Study 2 were lower on average than those in Study 1 despite the similar rank-orders of emotions. This may be due to the worsening economic outlook over the course of data collection (2007 Study 1; 2009 Study 2). Although this is speculative, participants might have implemented a tighter budget in many aspects of their lives, and generalized this conservative budget to their WTP judgments for emotions.
} 
Table 2 Study 2: means and standard deviations of willingness to pay judgments for emotional experiences among British and Hong Kong Chinese participants (ranked from highest to lowest in the UK)

\begin{tabular}{|c|c|c|c|c|c|c|c|}
\hline & \multicolumn{4}{|c|}{ British WTP (in GBP) } & \multicolumn{3}{|c|}{ Hong Kong Chinese WTP (in HKD) } \\
\hline & $M$ & SD & Rank & Study 1 Rank & $M$ & SD & Rank \\
\hline Love & 71.95 & 50.65 & 1 & 1 & 880.44 & 501.83 & 1 \\
\hline Happiness & 60.49 & 49.84 & 2 & 2 & 613.04 & 410.75 & 8 \\
\hline Sadness & 54.15 & 46.31 & 3 & 3 & 719.57 & 452.95 & 4 \\
\hline Delight & 51.71 & 45.66 & 4 & & 532.61 & 395.56 & 10 \\
\hline Fear & 51.22 & 47.97 & 5 & 9 & 645.65 & 433.95 & 7 \\
\hline Embarrassment & 44.88 & 38.41 & 6 & 6 & 773.91 & 485.54 & 3 \\
\hline Regret & 40.24 & 40.09 & 7 & 8 & 823.91 & 527.12 & 2 \\
\hline Pride & 38.78 & 30.84 & 8 & 11 & 652.17 & 459.83 & 6 \\
\hline Excitement & 38.05 & 35.09 & 9 & & 486.96 & 376.89 & 11 \\
\hline Calm & 33.41 & 32.99 & 10 & & 343.48 & 290.33 & 13 \\
\hline Anger & 32.68 & 35.22 & 11 & 10 & 413.04 & 363.08 & 12 \\
\hline Frustration & 32.44 & 25.38 & 12 & & 663.04 & 459.64 & 5 \\
\hline Enthusiasm & 26.58 & 21.63 & 13 & & 536.96 & 437.85 & 9 \\
\hline Boredom & 19.76 & 21.27 & 14 & & 282.61 & 280.72 & 14 \\
\hline Mean positive & 45.85 & 30.63 & & & 577.95 & 332.27 & \\
\hline Mean negative & 39.34 & 27.55 & & & 617.39 & 320.81 & \\
\hline
\end{tabular}

Positive emotions $=$ WTP to re-create; negative emotions $=$ WTP to avoid

among the British sample, it was ranked only 8th among Hong Kong Chinese, and the latter ranked regret 2nd, whereas British participants ranked it 7 th.

To facilitate cross-cultural comparisons we computed mean-centered scores for each emotion within each sample using raw scale responses (i.e., 1-15 rather than the currency amounts). These country-specific mean-centered scores were submitted to a 2 (Culture: British, Hong Kong Chinese) by 14 (Emotions: Love, Happiness, Sadness, Delight, Fear, Embarrassment, Regret, Pride, Excitement, Calm, Anger, Frustration, Enthusiasm, Boredom) multivariate ANOVA. As predicted, WTP judgments were not equivalent for all emotions across cultures, $V=0.412, F(13,73)=3.93, p<.001$ Pillai's trace. To explore which emotions British and Hong Kong Chinese participants differed on, we referred to the univariate outputs from the MANOVA (Table 3). The positive emotions of happiness, delight and calm all received higher relative WTP offers among British than Hong Kong Chinese participants. The opposite was the case for the negative emotions of embarrassment, regret and frustration. No other emotions differed significantly across samples.

To examine this apparent difference in WTP for positive versus negative emotions we collapsed the mean-centered scores for all positive and negative emotions and conducted a 2 (Culture: British, Hong Kong Chinese) by 2 (Valence: positive, negative) mixed-factorial ANOVA with repeated measures on the second factor. There was no main effect of Culture because the scores were identical due to the within country mean-centering, and no main effect of valence, $F(1,85)=0.31, p<.58$. However, there was a significant interaction, $F(1,85)=5.05, p<.03$. Controlling for overall tendencies within country, follow-up independent t-tests found that British participants were willing to pay significantly more $(M=0.33)$ than Hong Kong Chinese $(M=-0.20)$ to experience positive emotions, 
Table 3 Study 2: means and standard deviations of mean-centered willingness to pay judgments (from 1 to 15) among British and Hong Kong Chinese participants (ranked from highest to lowest in the UK)

\begin{tabular}{|c|c|c|c|c|c|c|}
\hline & \multicolumn{2}{|l|}{ British } & \multicolumn{2}{|c|}{ Hong Kong Chinese } & \multicolumn{2}{|c|}{ Difference between samples } \\
\hline & $M$ & $\mathrm{SD}$ & $M$ & SD & $F(1,85)$ & $p$ \\
\hline Love & 2.94 & 3.14 & 2.83 & 3.25 & 0.03 & .876 \\
\hline Happiness & 1.79 & 3.03 & 0.15 & 2.26 & 8.23 & .005 \\
\hline Sadness & 1.16 & 2.93 & 1.22 & 3.42 & 0.01 & .926 \\
\hline Delight & 0.91 & 2.58 & -0.65 & 2.23 & 9.18 & .003 \\
\hline Fear & 0.86 & 3.92 & 0.48 & 3.24 & 0.25 & .620 \\
\hline Embarrassment & 0.23 & 2.47 & 1.76 & 3.26 & 6.00 & .016 \\
\hline Regret & -0.24 & 2.62 & 2.26 & 3.77 & 12.57 & .001 \\
\hline Pride & -0.38 & 2.10 & 0.55 & 2.65 & 3.20 & .077 \\
\hline Excitement & -0.45 & 2.53 & -1.11 & 2.94 & 1.21 & .273 \\
\hline Calm & -0.92 & 2.96 & -2.54 & 2.61 & 7.39 & .008 \\
\hline Anger & -0.99 & 2.04 & -1.85 & 2.81 & 2.59 & .112 \\
\hline Frustration & -1.02 & 1.88 & 0.65 & 3.02 & 9.28 & .003 \\
\hline Enthusiasm & -1.60 & 2.08 & -0.61 & 3.15 & 2.94 & .090 \\
\hline Boredom & -2.28 & 2.74 & -3.15 & 2.53 & 2.35 & .129 \\
\hline Mean positive ${ }^{a}$ & 0.33 & 0.97 & -0.20 & 1.18 & 5.05 & .027 \\
\hline Mean negative & -0.33 & 0.97 & 0.20 & 1.18 & 5.05 & .027 \\
\hline
\end{tabular}

Positive emotions $=$ WTP to re-create; negative emotions $=$ WTP to avoid

${ }^{a}$ Due to mean-centering of WTP judgments, within-country mean positive and mean negative deviate from the common grand-mean by the same absolute amount, but with opposite signs

$t(85)=2.25, p<.027, d=0.49$, but significantly less to avoid negative emotions, $M \mathrm{~s}=-0.33 ; 0.20, t(85)=2.25, p<.027, d=0.49$.

\subsection{Discussion}

Study 2 largely replicated the value-based ranking of emotions and the positivity bias on WTP judgments obtained in Study 1 in another group of British undergraduates using a greater repertoire of emotion items. The study also demonstrated differences in evaluation of emotions across members of different cultural groups by exploring the WTP judgments of Hong Kong Chinese undergraduates.

Participants from both groups were willing to spend most on experiencing love (see also Sommers 1984a), consistent with the notion of a strong need to establish and sustain caring social ties (Baumeister and Leary 1995). Interestingly, both Chinese and British participants were not willing to pay much to experience pride compared to other emotional experiences, which is in line with the universal hesitancy to express pride found by Sommers (1984a).

Consistent with the findings of Eid and Diener (2001), cross-cultural comparisons of emotions suggested that British participants were willing to pay more to experience positive emotions and avoid negative ones than Hong Kong Chinese participants. When controlling for each culture's tendency to consistently pay more (or less) for emotions, significant cross-cultural differences in WTP judgments were found in six out of fourteen items, and two additional items showed marginally significant differences. British 
participants were willing to pay significantly more than their Hong Kong Chinese counterparts for experiencing pleasant emotions such as happiness, delight, and calm, whereas Hong Kong participants were willing to pay significantly more to avoid the unpleasant emotions of regret, embarrassment and frustration. Unlike their British counterparts Hong King Chinese participants did not show a preference for experiencing positive over avoiding experiences of negative emotions, consistent with findings that they take both into account when evaluating experiences (Wirtz et al. 2009). Results are also consistent with differences in self-regulatory focus of the two cultures (A.Y. Lee et al. 2000), with British participants exhibiting a more approach-oriented regulatory focus for positive emotions and Hong Kong Chinese participants exhibiting a more avoidance-oriented regulatory focus. For instance, the finding that Hong Kong Chinese were prepared to pay more than British participants in mitigating embarrassment is consistent with the functional importance of aversive self-conscious emotions in upholding social norms among members of collectivistic cultures (Tangney 1999). Thus, beneath the global patterns of positivenegative emotions we observed a more nuanced pattern of specific emotions that are more or less valued in different cultures.

\section{General Discussion}

Emotions are embedded in the goods and services we consume daily. We pay to have fun at the fairground and avoid frustration by recruiting someone to help complete tax returns. The current research attempted to quantify the value that people put on different emotions unmediated by the goods and services they purchase. In Study 1 British participants indicated how much they would be willing to pay to experience positive, and avoid negative, emotions. Study 2 examined the robustness of the approach across a broader range of emotions and explored potential cultural differences in these valuations between British and Hong Kong Chinese students.

Consistent with previous research, participants were willing to pay most to experience the feeling of love, reflecting a broadly held belief that satisfactory interpersonal relationships are a key component of happiness (e.g., Furnham and Cheng 2000; D. Y. Lee et al. 2000; Ryff 1989). Our WTP approach also highlighted cross-cultural differences: British participants prized experiencing positive emotions over avoiding unpleasant emotions, whereas Hong Kong Chinese participants demonstrated no such difference. This divergence is consistent with differences in prevalent self-regulatory focus of the two cultures (A. Y. Lee et al. 2000). Taken together the current studies showed that participants are able to use a WTP approach to differentiate their preference for emotions and that culture plays a predictable role in this process.

\subsection{Emotion Preferences, Cultural Norms and Affective Forecasting}

We observed a clear pattern of emotional preferences that was moderated by cultural factors: British participants showed a strong preference to experience positive emotions, whereas Hong Kong Chinese participants showed an equal preference for experiencing positive emotions and avoiding negative emotions. To what extent might such preferences be adaptive? If we consider whether participants are engaging in an affective forecasting error, it needs to be clear whether it would objectively be better for overall life satisfaction to experience positive events and emotions, but not negative events and emotions. Unfortunately, the literature so far has resulted in somewhat contradictory conclusions, 
with some studies finding that both types of emotion influenced well-being equally (Suh et al. 1996, 1998, Study 1), other studies suggesting a greater influence of positive factors (Suh et al. 1998, Study 2; Kuppens et al. 2008), and yet others suggesting a more pronounced impact of negative factors (Oishi et al. 2007).

Further, rather than looking at the frequency of positive and negative emotions separately, some have proposed to look at the relative ratio of the two, conceptualized as hedonic balance (Schimmack et al. 2002b), and cultures differ in the extent to which they consider it possible to experience both types of emotions at the same time (Schimmack et al. 2002a). Given the complexity of the issue, it is at present difficult to say whether participants' WTP judgments are adaptive in accurately predicting which emotions might be important to pursue. What is clear, however, is that these preferences are strongly shaped by cultural norms.

Our findings are in line with earlier work suggesting that people have an implicit understanding of what emotions they are expected to feel; in Western cultures, this involves coming across as outgoing, happy and confident, whereas in Eastern cultures negative emotions such as guilt and shame are more normative (Eid and Diener 2001; Sommers 1984a). Perhaps due to this focus on positive affect in individualistic cultures, and the propensity to notice and recall them proficiently (Oishi 2002; Wirtz et al. 2009), these are also the emotions that come to mind when thinking of the ideal case scenario. Indeed, Robinson and Clore (2002a) showed that when reporting on emotional experiences, people can either base their recollection on episodic memory, that is, examples of recent occurrences, or on semantic memory, namely general knowledge structures and beliefs. When questioned regarding longer time frames, or how they feel in general, people are more likely to rely on beliefs rather than on specific examples of emotional experiences. Our paradigm involved indicating the desirability of emotions in the abstract, and giving a global rather than contextualized judgment. Thus, Robinson and Clore's (2002b) accessibility model would predict that for such general judgments people should draw on beliefs, norms and expectations, which is precisely what we found for both countries.

\subsection{The Utility of the Willingness to Pay Approach}

The studies in this paper are only a first step in exploring how the WTP approach might aid our understanding of people's valuations of emotions. It is encouraging that responses complement earlier findings, but what added value does this approach offer over existing methods? We see at least two potential advantages, a theoretical one, and a practical one.

First, the WTP approach encourages people to think of emotions in terms of what they have to forego in order to experience or avoid them. Because money is a scarce resource for most people it constitutes a familiar unit by which to make trade-offs. The adoption of the WTP approach is therefore theoretically important because it is a closer approximation of the actual choices that people make in search of happiness in everyday life, compared to the responses given on the somewhat ambiguous response scales provided on Likert scales.

Second, the WTP approach may help applied efforts to understand the value of nonmarket goods. A good example is the UK's National Ecosystem Assessment (UK NEA 2011, see also Millennium Ecosystem Assessment 2005). The aim of ecosystem service assessments is to help policy makers understand the value of the natural environment regarding otherwise costly services. For instance, one way of understanding the importance of insects for pollinating crops is to derive a monetary estimate of what it would cost to pay someone to pollinate them by hand (i.e. the opportunity cost). Importantly, the UK NEA also argues that one of the potential services offered by natural environments is emotion 
regulation, because they can help combat negative emotions and enhance positive ones (UK NEA 2011, Chap. 23). Although a growing body of work supports this claim (e.g. Maas et al. 2009; Mitchell and Popham 2008) it remains unclear how objectively "valuable" this function is. We suggest that the WTP approach offers a potential way of measuring these benefits. These values could then feed into habitat protection decisions alongside other ecosystem services.

\subsection{Issues and Limitations}

Both the methodology of the current studies and the general approach may raise a number of potential issues. First, we used small convenience samples of students and are not suggesting that the values we present here are representative of those that might be expressed within these populations as a whole, especially given the small to medium effect sizes. The purpose of the current research was simply to explore whether respondents could use the measures by comparing whether their answers were consistent with those elicited via alternative methods in earlier research. As such, this was a test case, and further research will need to develop the technique and examine its potential for use with larger sample sizes, participants with different demographic characteristics (e.g., in terms of age groups and socio-economic statuses), and in different contexts.

Second, we presume cultural differences regarding independent or interdependent selfconstruals in Study 2, but did not assess them directly. Doing so would allow testing the mechanism behind the differences in WTP judgments of Hong Kong Chinese and British participants, and once established, cultural influences on emotional preference could illuminate their functions, and how people set emotional goals.

Third, although using the same language for the questionnaire with both English and Hong Kong students in Study 2 provided several advantages (e.g. linguistic continuity and lack of translation issues) we recognize that this may have masked some differences. Recent research suggests, for instance, that multicultural participants may employ different theories of subjective well-being when primed with different cultural mindsets, and that language can exert such a priming effect (Tam et al. 2012). If true of the current findings it would suggest a conservative estimate of cross-cultural differences and thus use of mother tongue (or first language) questionnaires may have shown stronger effects. We therefore recognize the importance of using mother tongue questionnaires in future while also acknowledging the unique opportunity the current samples offered in terms of questionnaire standardization.

In terms of more theoretical issues, extensive discussions of the limitations of the WTP approach already exist (Bateman et al. 2002). For present purposes we highlight four potential challenges for future research using a WTP approach to value emotions. First, psychologists have discussed the idea of "taboo tradeoff", i.e. the notion that emotions may be sacred and should not to be contaminated by secular money (Fiske and Tetlock 1997). Consequently, it might be possible that participants refuse to differentiate their preference for emotions via price-tags. Although possible, our findings did not suggest such a problem, but we recognize that these issues may become more important for other populations or in other contexts.

Second, rather than reflecting the importance of specific emotions, a willingness to pay approach may be picking up difficulties in perceived emotion regulation. A person who has trouble controlling their own anger, for instance, may be willing to pay more to avoid being angry than someone who is more in control of this emotion. Along similar lines individuals may be willing to pay more for emotions they experience rarely (i.e., a scarcity effect) and/or 
less for emotions they experience often (i.e., a satiation effect). Thus, future research needs to examine the extent to which participants might use the WTP approach as a way of expressing relative emotional importance in general rather than idiosyncratic emotional goals.

Third, there may be an essential asymmetry between experiencing positive emotions, which people interpret as also reflecting the absence of negative emotions, and avoiding negative emotions, which could imply neutrality rather than the presence of positive emotions. Thus, higher WTP estimates for positive emotions may reflect a belief in a double benefit. Although possible, this does not explain why Hong Kong Chinese participants did not show the same positivity bias as UK participants, or why the latter were willing to pay substantially more to avoid negative emotions such as sadness and fear than positive emotions such as pride or excitement. Nevertheless, a more direct exploration of people's underlying assumptions using a WTP paradigm is warranted.

Finally, our instructions did not specify how participants should achieve or avoid the emotional experiences through their WTP estimates. It was also uncertain, for instance, whether the WTP estimate of avoiding an emotion refers to paying to undo the negative consequences of an event (e.g., paying a parking fine quickly before the cost rises), paying to mollify distress (e.g., buying a drink because one is angry about being fined), or paying to sidestep the event altogether (e.g., paying for a taxi and avoid being fined). Similar uncertainty exists for positive emotions. Thus, future studies may be fine-tuned to be more sensitive to participants' different stages of mood regulation (Gross 2001), and compare their different attempts to experience and avoiding emotions accordingly.

\subsection{Conclusions}

We developed a novel WTP approach of estimating preferences for emotional experiences across cultures. Because such an approach assumes opportunity costs associated with each preference and makes use of cardinal properties of money, resulting rank orders may capture preferences more closely than traditional Likert scales. Further, the WTP paradigm provides an ecologically valid measure to assess emotions because the same unit is used as for other consumption behaviors occurring in daily life. Our data suggest that participants intuitively understood the logic behind this approach and had no difficulty in applying it to their own context. Thus, by putting price-tags on emotions we might come closer to understanding the value of human experience in order to aid policies aimed at enhancing well-being.

Acknowledgments This research was supported by RES-000-22-4453 from the Economic and Social Research Council to S.S. M.W. works at the ECEHH which is supported by the European Regional Development Fund and the European Social Fund Convergence Programme for Cornwall and the Isles of Scilly. We thank Claudia Schoenbohm for help with the data collection of Study 1, and Kevin Tam and Melody Chao for their helpful comments on an earlier draft of this paper.

Open Access This article is distributed under the terms of the Creative Commons Attribution License which permits any use, distribution, and reproduction in any medium, provided the original author(s) and the source are credited.

\section{References}

Adamowicz, W. L., Louviere, J., \& Williams, M. (1994). Combining stated preferences and revealed preference methods for valuing environmental amenities. Journal of Environmental Economics and Management, 26, 271-292. 
Aristotle. (1988). Nicomachean ethics (W. D. Ross, J. W. Ackril, \& J. O. Urmson, Trans.). Oxford: Oxford University Press. (Original work published ca. 350 B.C.).

Augustine, A. A., Hemenover, S. H., Larsen, R. J., \& Shulman, T. E. (2010). Composition and consistency of the desired affective state: The role of personality and motivation. Motivation and Emotion, 34, $133-143$.

Bateman, I. J., Carson, R. T., Day, B., Hanemann, W. M., Hanley, N., Hett, T., et al. (2002). Economic valuation with stated preference techniques: A manual. Cheltenham: Elgar.

Baumeister, R. F., Bratslavsky, E., Finkenauer, C., \& Vohs, K. D. (2001). Bad is stronger than good. Review of General Psychology, 5, 323-370.

Baumeister, R. F., \& Leary, M. R. (1995). The need to belong: Desire for interpersonal attachments as a fundamental human motivation. Psychological Bulletin, 117, 497-529.

Bodenhausen, G. V., Sheppard, L. A., \& Kramer, G. P. (1994). Negative affect and social judgment: The differential impact of anger and sadness. European Journal of Social Psychology, 24, 45-62.

Cacioppo, J. T., Gardner, W. L., \& Berntson, G. G. (1999). The affect system has parallel and integrative processing components: Form follows function. Journal of Personality and Social Psychology, 76, 839-855.

Canova, L., Rattazzi, A. M. M., \& Webley, P. (2005). Hierarchical structure of saving motives. Journal of Economic Psychology, 26, 21-34.

Clore, G. L., \& Huntsinger, J. R. (2007). How emotions inform judgment and regulate thought. Trends in Cognitive Sciences, 11, 393-399.

Cohen, S., \& Pressman, S. D. (2006). Positive affect and health. Current Directions in Psychological Science, $15,122-125$.

Danner, D. D., Snowdon, D. A., \& Friesen, W. V. (2001). Positive emotions in early life and longevity: Findings from the nun study. Journal of Personality and Social Psychology, 80, 804-813.

Davidson, R. J., Jackson, D. C., \& Kalin, N. H. (2000). Emotion, plasticity, context and regulation: Perspectives from affective neuroscience. Psychological Bulletin, 126, 890-906.

Deci, E. L., \& Ryan, R. M. (2000). The "What" and "Why" of goal pursuits: Human needs and the selfdetermination of behavior. Psychological Inquiry, 11, 227-268.

Diener, M. L., \& Lucas, R. E. (2004). Adults' desires for children's emotions across 48 countries: Associations with individual and national characteristics. Journal of Cross- Cultural Psychology, 35, $525-547$.

Diener, E., Suh, E. M., Lucas, R. E., \& Smith, H. L. (1999). Subjective well-being: Three decades of progress. Psychological Review, 125, 276-302.

Dolan, P., \& Kahneman, D. (2008). Interpretations of utility and their implications for the valuation of health. The Economic Journal, 118, 215-234.

Eid, M., \& Diener, E. (2001). Norms for experiencing emotions in different cultures: Inter- and intranational differences. Journal of Personality and Social Psychology, 81, 869-885.

Fiske, A. P., \& Tetlock, P. E. (1997). Taboo trade-offs: Reactions to transactions that transgress spheres of justice. Political Psychology, 18, 255-297.

Fredrickson, B. L. (2004). The broaden-and-build theory of positive emotions. Philosophical Transactions of the Royal Society B: Biological Sciences, 359, 1367-1378.

Furnham, A., \& Cheng, H. (2000). Lay theories of happiness. Journal of Happiness Studies, 1, $227-246$.

Gross, J. J. (2001). Emotion regulation in adulthood: Timing is everything. Current Directions in Psychological Science, 10, 214-219.

Kahneman, D. (2000). Experienced utility and objective happiness: A moment-based approach. In D. Kahneman \& A. Tversky (Eds.), Choices, values and frames (pp. 673-692). Cambridge: Cambridge University Press.

Kahneman, D., Krueger, A. B., Schkade, D. A., Schwarz, N., \& Stone, A. A. (2004). A survey method for characterizing daily life experience: The day reconstruction method (DRM). Science, 306, 1776-1780.

Kahneman, D., Ritov, I., Jacowitz, K. E., \& Grant, P. (1993). Stated willingness to pay for public goods: A psychological perspective. Psychological Science, 4, 310-315.

Kahneman, D., \& Sugden, R. (2005). Experienced utility as a standard of policy evaluation. Environmental \& Resource Economics, 32, 161-181.

Kahneman, D., \& Tversky, A. (1979). Prospect theory: An analysis of decision under risk. Econometrica, 47, 263-292.

Kampfe, N., \& Mitte, K. (2009). What you wish is what you get? The meaning of individual variability in desired affect and affective discrepancy. Journal of Research in Personality, 43, 409-418.

Kuppens, P., Realo, A., \& Diener, E. (2008). The role of positive and negative emotions in life satisfaction judgments across nations. Journal of Personality and Social Psychology, 95, 66-75.

Larsen, R. J. (2000). Toward a science of mood regulation. Psychological Inquiry, 11, 129-141. 
Lee, A. Y., Aaker, J. L., \& Gardner, W. L. (2000). The pleasures and pains of distinct self-construals: The role of interdependence in regulatory focus. Journal of Personality and Social Psychology, 78, 1122-1134.

Lee, D. Y., Park, S. H., Uhlemann, M. R., \& Patsult, P. (2000). What makes you happy? A comparison of self-reported criteria of happiness between two cultures. Social Indicators Research, 50, 351-362.

Maas, J., Verheij, R. A., de Vries, S., Spreeuwenberg, P., Schellevis, F. G., \& Groenewegen, P. P. (2009). Morbidity is related to a green living environment. Journal of Epidemiology and Community Health, 63, 967-973.

Millennium Ecosystem Assessment. (2005). Ecosystems and human well-being: Synthesis. Washington, DC: Island Press.

Mitchell, R., \& Popham, F. (2008). Effect of exposure to natural environment on health inequalities: an observational population study. Lancet, 372, 1655-1660.

Oishi, S. (2002). The experiencing and remembering of well-being: A cross-cultural analysis. Personality and Social Psychology Bulletin, 28, 1398-1406.

Oishi, S., Diener, E., Choi, D. W., Kim-Prieto, C., \& Choi, I. (2007). The dynamics of daily events and wellbeing across cultures: When less is more. Journal of Personality and Social Psychology, 93, 685-698.

Ortony, A., Clore, G. L., \& Collins, A. (1988). The cognitive structure of emotions. New York: Cambridge University Press.

Reisenzein, R. (1994). Pleasure-arousal theory and the intensity of emotions. Journal of Personality and Social Psychology, 6, 525-539.

Robinson, M. D., \& Clore, G. L. (2002a). Episodic and semantic knowledge in emotional self-report: Evidence for two judgment processes. Journal of Personality and Social Psychology, 83, 663-677.

Robinson, M. D., \& Clore, G. L. (2002b). Belief and feeling: Evidence for an accessibility model of emotional self-report. Psychological Bulletin, 128, 934-960.

Roseman, I. J., Spindel, M. S., \& Jose, P. E. (1990). Appraisals of emotion-eliciting events: Testing a theory of discrete emotions. Journal of Personality and Social Psychology, 59, 899-915.

Russell, J. A. (1980). A circumplex model of affect. Journal of Personality and Social Psychology, 39, 1161-1178.

Rusting, C. L., \& Larsen, R. J. (1995). Moods as sources of stimulation: Relationships between personality and desired mood states. Personality and Individual Differences, 18, 321-329.

Ryff, C. D. (1989). Happiness is everything, or is it? Explorations on the meaning of psychological wellbeing. Journal of Personality and Social Psychology, 57, 1069-1081.

Scherer, K. R., Schorr, A., \& Johnstone, T. (Eds.). (2001). Appraisal processes in emotion: Theory, methods, research. Oxford: Oxford University Press.

Schimmack, U. (2005). Attentional interference effects of emotional pictures: Threat, negativity, or arousal? Emotion, 5, 55-66.

Schimmack, U., Oishi, S., \& Diener, E. (2002a). Cultural influences on the relation between pleasant emotions and unpleasant emotions: Asian dialectic philosophies or individualism-collectivism? Cognition and Emotion, 16, 705-719.

Schimmack, U., Radhakrishnan, P., Oishi, S., Dzokoto, V., \& Ahadi, S. (2002b). Culture, personality, and subjective well-being: Integrating process models of life satisfaction. Journal of Personality and Social Psychology, 82, 582-593.

Seery, M. D., Holman, E. A., \& Silber, R. C. (2010). Whatever does not kill us: Cumulative lifetime adversity, vulnerability, and resilience. Journal of Personality and Social Psychology, 99, 1025-1041.

Sommers, S. (1984a). Adults evaluating their emotions: A cross-cultural perspective. In C. Z. Malatesta \& C. E. Izard (Eds.), Emotion in adult development (pp. 319-338). Beverly Hills, CA: Sage.

Sommers, S. (1984b). Reported emotions and conventions of emotionality among college students. Journal of Personality and Social Psychology, 46, 207-215.

Suh, E., Diener, E., \& Fujita, F. (1996). Events and subjective well-being: Only recent events matter. Journal of Personality and Social Psychology, 70, 1091-1102.

Suh, E., Diener, E., Oishi, S., \& Triandis, H. C. (1998). The shifting basis of life satisfaction judgments across culture: Emotions versus norms. Journal of Personality and Social Psychology, 74, 482-493.

Tam, K. P., Lau, H. P. B., \& Jiang, D. (2012). Culture and subjective well-being: A dynamic constructivist view. Journal of Cross-Cultural Psychology, 43, 23-31.

Tangney, J. P. (1999). The self-conscious emotions: Shame, guilt, embarrassment and pride. In T. Dalgleish \& M. J. Power (Eds.), Handbook of cognition and emotion (pp. 541-568). New York: Wiley.

Tsai, J. L., Knutson, B., \& Fung, H. H. (2006). Cultural variation in affect valuation. Journal of Personality and Social Psychology, 90, 288-307. 
Tsai, J. L., Miao, F. F., Seppala, E., Fung, H. H., \& Yeung, D. Y. (2007). Influence and adjustment goals: Sources of cultural differences in ideal affect. Journal of Personality and Social Psychology, 92, 1102-1117.

UK National Ecosystem Assessment (UK-NEA). (2011). Understanding nature's value to society. Oxford: Information Press.

United Nations Statistics Division (2009, September). Purchasing Power Parities (PPP) Conversion Factor, Local currency unit to international dollar. Retrieved January 3, 2010 from http://data.un.org/ Data.aspx?d=MDG\&f=seriesRowID:699.

Wilson, T. D., \& Gilbert, D. (2003). Affective forecasting. Advances in Experimental Social Psychology, 35, 345-411.

Wirtz, D., Chiu, C., Diener, E., \& Oishi, S. (2009). What constitutes a good life? Cultural differences in the role of positive and negative affect in subjective well-being. Journal of Personality, 77, 1167-1195. 